\title{
Detection and monitoring prostate specific antigen using nanotechnology approaches to biosensing
}

\author{
Grant Perry, Fernando Cortezon-Tamarit ( $₫)$, Sofia I. Pascu ( $₫)$ \\ Department of Chemistry, University of Bath, Claverton Down, Bath BA2 7AY, UK
}

(C) The Author(s) 2019. This article is published with open access at link.springer.com and journal.hep.com.cn

\begin{abstract}
Prostate cancer has a high incidence in men and remains the second cause of mortality due to cancer worldwide. As the development of the disease is greatly correlated to age, the identification of novel detection methods reliable, efficient, and cost effective is a matter of significant importance in the ageing population of western societies. The detection of the prostate specific antigen (PSA) in blood samples has been the preferred method for the detection and monitoring of prostate cancer over the past decades. Despite the indications against its use in massive population screening, PSA still remains the best studied biomarker for prostate cancer and the detection of its different forms and incorporation in multiplexed designs with other biomarkers still remains a highly valuable indicator in the theranostics of prostate cancer. The latest developments in the use of nanomaterials towards the construction of PSA biosensors are reviewed hereby. The incorporation of gold nanoparticles, silica nanoparticles and graphene nanostructures to biosensing devices has represented a big leap forward in terms of sensitivity, stability and miniaturization. Both electrochemical and optical detection methods for the detection of PSA will be reviewed herein.
\end{abstract}

Keywords biosensing, immunosensor, PSA, prostate cancer, fluorescence

\section{Introduction}

Cancer constitutes a major health concern, as an example, 3.9 million new cancer cases were diagnosed only in Europe with 1.9 million deaths [1]. Furthermore, annual medical costs for care related to cancer only were estimated at $\$ 219$ billion in the US and $€ 126$ billion in Europe [2]

Received November 26, 2018; accepted March 29, 2019

E-mails: s.pascu@bath.ac.uk (Pascu S I),

fctc20@bath.ac.uk (Cortezon-Tamarit F) according to data collected during the past decade (2008, 2009 respectively for US and EU data). Prostate cancer is one of the main contributors in number of cases and the second cause of cancer mortality among men after lung cancer adding up for ca. $20 \%$ of all new cancer cases and ca. $10 \%$ to the overall mortality (Fig. 1) $[1,3]$. The development of prostate cancer $(\mathrm{PCa})$ is strongly correlated to age hence the detection and monitoring of the disease is a matter of special importance in ageing western populations. Consequently, the development of biosensors able to accurately detect biomarkers in fluids has bloomed in the past decades following the clinical drive to design

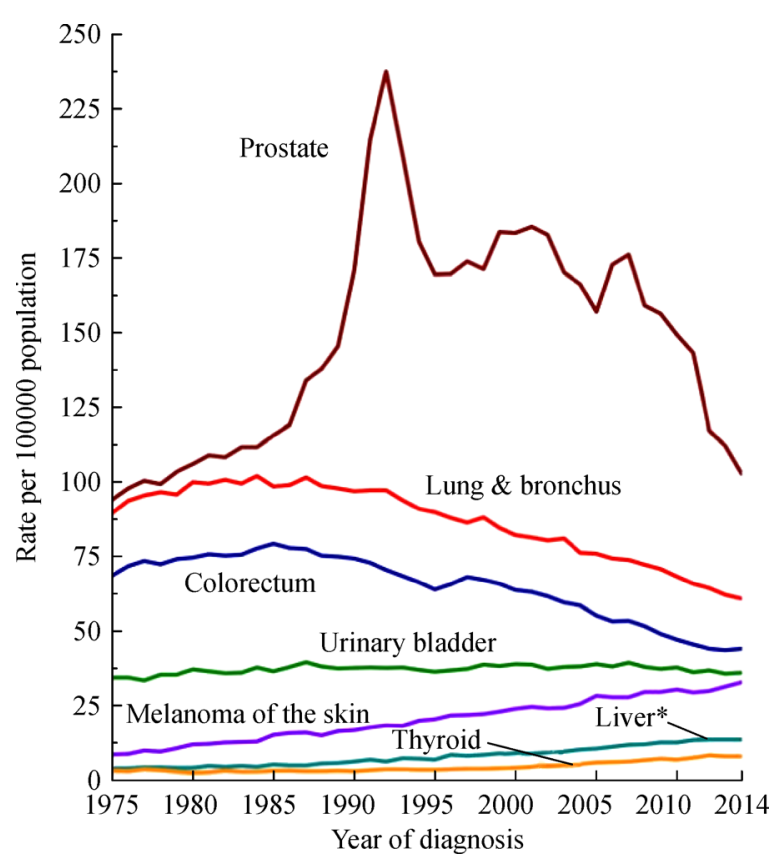

Fig. 1 Incidence rates of different cancers in men in the United States during the 1975 to 2014 period. The spike in prostate cancer cases during the mid-1990s was produced due to the introduction of PSA screening. Adapted from Ref. [3] with permission from John Wiley and sons, copyright (2018) 
personalized medical treatments capable of reducing patient exposure and expenditure on unnecessary tests and medication.

The prognosis of most cancers is related to an early diagnosis hence the identification of biomarkers, development of novel diagnostic tools and improvement of detection thresholds is of capital importance. In this sense, the intense research in nanotechnology in the past two decades has attracted considerable attention for its application in medical diagnosis [4], imaging or treatment [5]. The most common methods of prostate cancer diagnosis to date involve physical examination (digital rectal examination), trans-rectal ultrasound or biopsies of the prostate [6]. These are all invasive methods presenting problems in patient's acceptability and do not necessarily guarantee an accurate answer to the presence/location of the disease. The most used biomarker in the evaluation of prostate cancer has been the prostate specific antigen (PSA) which is present in small quantities in the serum of men with healthy prostates. Generally, PSA concentrations greater than $4 \mathrm{ng} \cdot \mathrm{mL}^{-1}$ in blood could indicate the first stages of prostate cancer, with levels greater than $10 \mathrm{ng} \cdot \mathrm{mL}^{-1}$ being considered a clear indicative of this disease onset. However, there has been some controversy in the use of PSA alone as a screening or decision-making tool due to the risk of overdiagnosis and overtreatment. This has led the US Preventive Services Task Force to recommend against mass screening of men population using PSA regardless of the age and only employ it as follow up tool after treatment [7]. Consequently, the identification of novel prostate cancer biomarkers to use in conjugation with PSA is vital for the correct identification of the presence and nature of the disease in a wide range of patients. Other biomarkers such as the prostate specific membrane antigen, prostate cancer gene 3 or $\alpha$-methylacylCoA racemase have been explored in research environments and biosensors multiplexed with PSA developed [810]. Despite the recommendations against the use of PSA as a massive screening tool, the prostate specific antigen still remains as the best studied PCa biomarker and can be a helpful asset in the detection and monitoring of the disease in particular cases, differentiating between its forms (free or complexed) or in conjunction with other biomarkers.

The application of nanomaterials to the production of prostate cancer biosensors can be broadly summarized considering the type of nanostructure used as a scaffold for the biosensing design (i.e., gold nanoparticles, carbon nanotubes, graphene, etc.) and by the nature of the physical response measured by the sensor, which generally results in the detection of optical or electrical signals. This work aims to provide an overview on the latest applications of nanostructured platforms to electronic or fluorescent biosensors for the detection of PSA in prostate cancer diagnosis setups.

The choice of biomarker is deliberate, given that the prostate specific antigen or human kallikrein related peptidase (KLK3) has been actively studied to date, and its detection applied clinically as a mean of detection and monitoring PCa post-diagnosis. PSA is an enzyme formed by a 237 residue glycoprotein (Fig. 2) of the kallikreine serine protease [11]. It is secreted by the prostate epithelium in healthy individuals as a component of semen. The catalytic active site is commonly free in seminal plasma but is complexed by endogenous inhibitors found in serum [12]. This raises the possibility that tests could discriminate between free and complexed PSA forms, which may help determine whether or not its production may be due to the presence of cancerous cells. This is generally achieved in research tools based on multiplexing biosensing devices currently in clinical use [13].

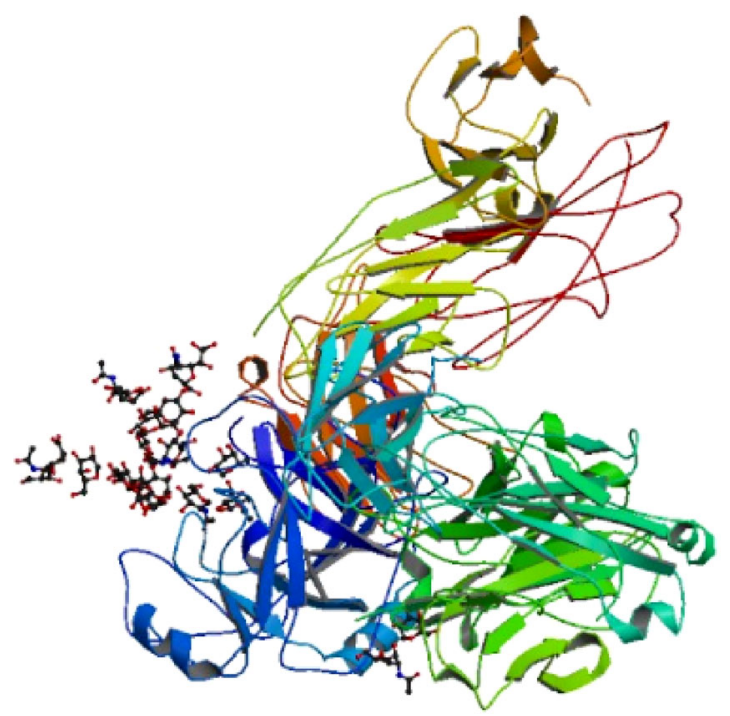

Fig. 2 Single crystal X-ray structure of the PSA enzyme in a sandwich with two antibodies. Image adapted from data on Ref. [12] deposited on the Protein Data Bank

\section{Overview of current PSA assays}

The current detection technique for PSA in clinical setups is by enzyme-linked-immunosorbent-assay (ELISA) $[14,15]$ and in less proportion by other detection methods such as radioimmunoassays [16]. ELISA uses a solidphase enzyme immunoassay to detect and quantify biomolecules (proteins, antibodies, hormones) specific to a disease/condition in order to confirm a diagnosis. Figure 3 shows schematically the steps involved in the direct ELISA, where antigens specific to a type of cancer are immobilized on the testing surface and recognized by antibodies conjugated with a reporter such as an enzyme. Generally, antibodies may bind to the specific antigens if these are present in the sample, then a signal produced by the reporter in the presence of a substrate is detected and quantified. However, this method presents a number of 


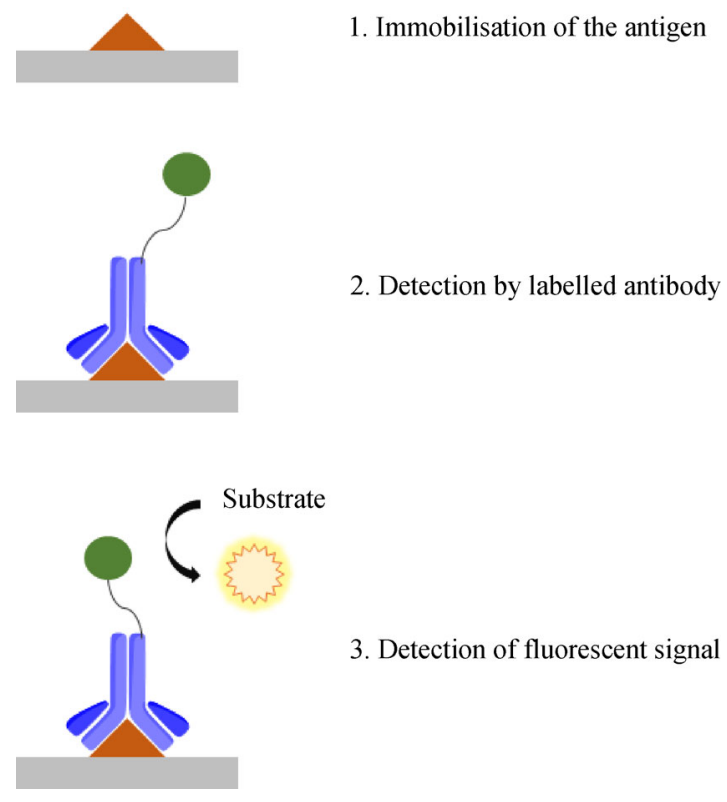

Fig. 3 Conceptual representation of direct ELISA

disadvantages (e.g., binding efficacy affected by modification of the primary antibody or lack of signal amplification) and currently the most applied methods are indirect ELISA or sandwich assays which allow for an increased versatility and sensitivity. ELISA, however, is both time consuming and expensive.

PSA is currently detected in clinical settings via ELISAbased protocols from blood samples, and as prostate cancer has a strong link between a rapid diagnosis and a good prognosis, a faster technique will be substantially beneficial. In addition, the majority of prostate cancer cases are diagnosed when the cancer is already established, and it would be advantageous to perform early detection for improved patient outcomes. The earlier the diagnosis takes place in the course of the disease, the better the prognosis. Research into aptamers (oligonucleotide or peptide molecules that bind to a specific target molecule) using
DNA [17] often conjugated with fluorescent dyes was undertaken. These sensors are more cost-effective, faster, simpler, and stable than those of ELISA [14].

Immunoassays are the current biochemical tests employed for general approaches to biomarker detection, and hence PSA detection. They measure the presence of a target molecule by concentration when detecting macromolecules, and by the use of antibody or antigen for small molecules. PSA is a biological protein molecule, and so antibodies are used in immunoassays for the detection of PSA. They come in many different test designs, such as sandwich assays [18], and the test design depends on the target molecule that is being detected. PSA has the ability to cleave certain DNA sequences [19], researchers have been able to identify these specific sequences and modify biosensors with them. These DNA sequences are aptamers, short, stable oligonucleotide sequences possessing high affinity and specificity for PSA [20]. Once the sensors are exposed to PSA, the DNA strand will be cleaved, and depending on the sensor design, it will measure an electrochemical change or observe fluorescence in the case of fluorescent labels, such as fluorescein isothiocyanate.

As an enzyme, PSA can react with and act by, cleaving, other biomolecules. The cleaved molecules can either become photoactive or be detected as a signal on/off in cyclic voltammetry measurements [22]. The electrochemical approach to PSA detection is very common in biosensors, and the performances of such biosensors depend on two important factors: the efficiency of the receptors on the sensors and the performance of the transduction method [23]. The photoactive approach [24] is less common in biosensors, but provides an unique and novel way of using visible light to detect PSA, hence may have use in biosensors in the general public. Figure 4 shows a simple reaction scheme for a photoactive based test, demonstrating the basic principles of PSA detection, an electrochemical test will show a reduced (signal off) or increased (signal on) current instead of measured fluorescence intensity.
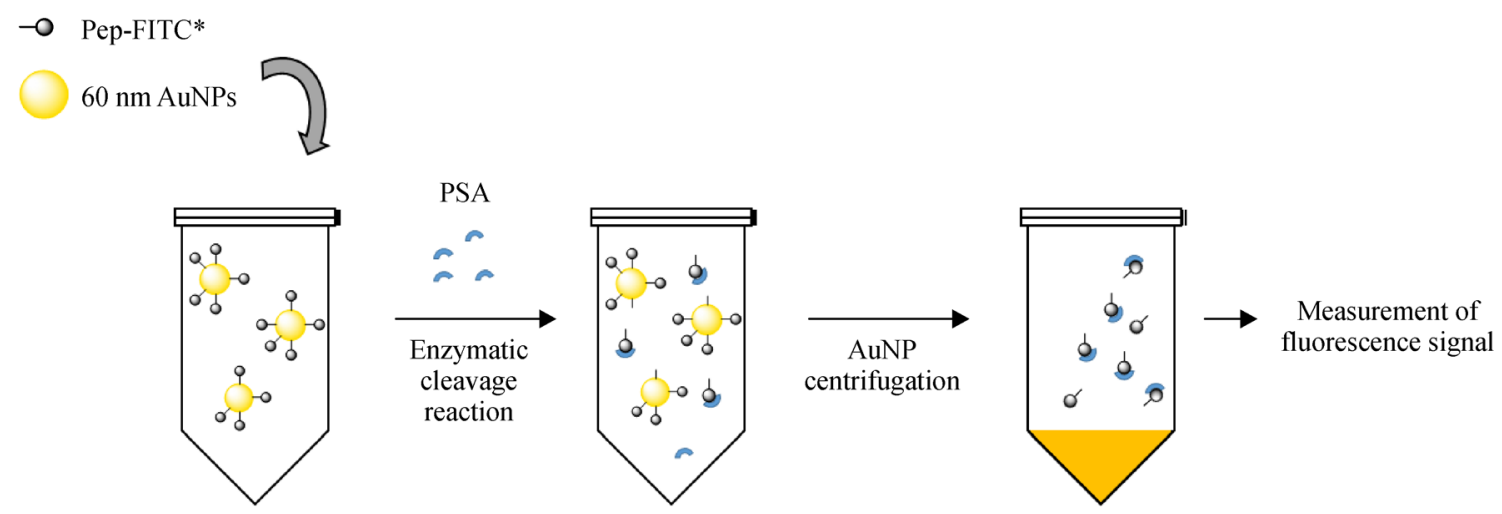

Fig. 4 Example of photoactive immunoassay for PSA. Adapted from Ref. [21]. AuNP: gold nanoparticles; *Including binding sequence to AuNP and enzymatic cleavage site 
Regarding the design of the sensors for PSA detection, several different approaches have been adopted, all with the goal of increasing sensitivity towards PSA. The most common design is a sandwich immunoassay [18], which is a dual site immunoassay where the target biomolecule is bound to the antibody site, then the labelled antibody is bound to the analyte. The problem facing current sensors for detecting PSA is sensitivity, given that a PSA concentration of just $4 \mathrm{ng} \cdot \mathrm{mL}^{-1}$ can indicate the presence of prostate cancer and hence developing sensors capable of reliable results at the $\mathrm{ng} \cdot \mathrm{mL}^{-1}$ concentration range is still the goal of many research groups. A proof of this interest is the commercialization of several PSA testing kits based on a sandwich immunofluorescent assays [25] such as the Brahms Kryptor total PSA test (sold by ThermoFisher) and which is based on the energy transfer and emission of an europium complex and a fluorescent protein. The introduction of a time delay in the detection allows for the removal of potential non-specific signals. In addition, the method does not require additional processing steps and has a wide measuring range with an automatic dilution system.

\section{Application of nanomaterials to the design of PSA biosensors}

Immunosensors based on nanotechnology design protocols, involving nanomaterials of controlled dimensions display a level of sensitivity towards their target unprecedented by other sensors thanks to the sensitivity enhancement [24]. Research into such sensors show that gold nanoparticles [26] and graphene nanostructures [27] are fundamental to these immunosensors. Hybrid immunosensors [28] show promise as they combine multiple attributes of two materials at a nanoscale level. Ongoing research is being undertaken into unique materials for novel biosensors [29]. Most commonly researched biosensors acting as aptasensors are impedimetric in nature, whereby they rely on the measure of the opposition to the flow of the current in electrochemical systems [30], measured by cyclic voltammetry. An alternative detection method is optical analysis in the case of inclusion of a fluorophore into the system design. This can be used solely in fluorescent sensors design or chemosensors, or in conjunction with an impedimetric sensor, emission is measured to determine if a target biomolecule is present [31].

Assessing the performance of the nano-aptasensors discussed in this report is generally performed by comparing the limit of detection (LOD) and linear range of the sensors. LOD is the lowest quantity of a substance that can be distinguished from the absence of that substance within a stated confidence limit. In the case of PSA, a concentration of the antigen above ca. $4.0 \mathrm{ng} \cdot \mathrm{mL}^{-1}$ is considered abnormal and as such sensors are required to be sensitive in this order of magnitude. The linear range is the range of the sensor, in which an input signal, such as the amount of PSA present, gives a linear function to an output signal, for example, an electric signal for electrochemical sensors or an optical response for fluorescent sensors. Below is a summary of a selection of such biosensors, which will be discussed based on the nature of the scaffold used in the design approaches.

\subsection{Gold NP-based aptasensors}

Gold nanoparticles-based aptasensors give a rapid and simple way of detecting PSA. With gold being relatively easy to work with, due to extensive previous research into gold nanoparticles, on the nanoscale and its ability to form complexes with proteins which can be cleaved by PSA, gold nanoparticles are fundamental to the assembly of sensors tailor-made for the PSA detection [32].

PSA cleaves certain proteins which arrive on its proximity. A sensor based on fluorescein isothiocyanate/ peptide-conjugated gold nanoparticle complexes [21], which fluoresces on PSA exposure can be used to detect PSA, is an example of an optical sensor. Using this method, the sensor was able to achieve a relatively good level of sensitivity, with a detection range of $10 \mathrm{pmol} \cdot \mathrm{L}^{-1}$ to $100 \mathrm{nmol} \cdot \mathrm{L}^{-1}$, however this sensor surpasses other gold nanoparticle aptasensors not in sensitivity but with respect to rapid and simple detection, which originates from the high enzymatic reactivity of the target protein attached to the gold nanoparticles. These properties are what is desired from a sensor applied in clinical or diagnostic applications, and with the possibility to modify this sensor for detection of other biomolecules, gives this design huge potential in clinical use [21].

The majority of biosensors utilize a signal-on method, as does this example, where gold nanoparticles are attached onto a gold electrode for impedimetric detection of PSA [32]. This example of a sensor showcases the effect of adding gold nanoparticles to a sensor system on sensitivity. Immobilizing DNA aptamers directly onto the surface of a gold electrode allows detection of biomarkers specific to the DNA aptamers, this simple systems gives a LOD of 60 of $\mathrm{ng} \cdot \mathrm{mL}^{-1}$. Comparing this to the sensor augmented with gold nanoparticles, shows a huge difference in performance regarding sensitivity, with the nanoparticle sensor showing a LOD of just $10 \mathrm{pg} \cdot \mathrm{mL}^{-1}$. The difference in surface structure is the reason behind the high performance of the gold nanoparticle sensor, mainly due to the increased surface area available to interact with PSA.

Gold nanoparticle-based sensors strive to tackle the sensitivity problems when detecting and quantifying PSA and a number of approaches of increased complexities. More advanced sensor designs may allow for a higher level of sensitivity to be reached. One such sensor uses gold nanoparticles encapsulated in polyamidoamine dendrimer and a layer of horseradish peroxidase linked aptamer in a 
sandwich format [33]. This is an example of a triple signal amplification strategy. The detection method for PSA for this sensor was the measurement of the electrocatalytic reduction of $\mathrm{H}_{2} \mathrm{O}_{2}$ in the presence of enzymatically oxidized thionine. The prepared immunosensor exhibited high sensitivity and a low LOD of $10 \mathrm{fg} \cdot \mathrm{mL}^{-1}$ and a linear range of $0.1 \mathrm{pg} \cdot \mathrm{mL}^{-1}$ to $909 \mathrm{ng} \cdot \mathrm{mL}^{-1}$, hence this immunosensor shows promise in application of PSA detection.

Research into new interdisciplinary fields always yields novel designs and sensors for detecting PSA are no exception. The use of nanoparticles as synthetic scaffolds now allow materials chemists to produce intricate nanostructures, enhancing the performance of such sensors. For example, one such sensor bears a resemblance to a hairbrush using gold nanostructure for detection towards PSA [34]. The nanostructure comprises of an array of nano-spindles applied onto the surface of a transducer, hence fabricating a signal-on electrochemical aptasensor. The nano-spindles, shown in Fig. 5, are long thin nanostructures, in this case comprising of gold. Sensor displayed a LOD of $50 \mathrm{pg} \cdot \mathrm{mL}^{-1}$ and a linear range of $0.125-128 \mathrm{ng} \cdot \mathrm{mL}^{-1}$, therefore biosensor exhibits potential for use in a clinical environment for detection of PSA.

\subsection{Graphene-based biosensors}

Graphene is a 2D material formulated as a honeycomb oneatom layer of $\mathrm{sp}^{2}$ carbon that possess unprecedented mechanical and conductive properties. Since the seminal work of Geim and Novoselov [35], new applications for graphene-based structures have grown exponentially including the field of biosensors where it can be used to increase conductivity and stability [36-38]. The affinity of gold nanoparticles to graphene in gold/graphene hybrid nanostructures has been proven to improve electrochemical immunosensors in terms of sensitivity [39]. Graphene may be essentially used as a signal amplifier in such PCa sensing applications, in order to achieve a lower limit of detection in immunosensors for detection of PSA.

The most common method in using graphene in the detection of PSA, is in a screen-printed form, in order to prepare an electrochemical biosensor. For example, the EN2 biomarker corresponding to prostate cancer is known to be a strong binder to a specific DNA sequence, hence taking advantage of this knowledge, the specific DNA sequence can be immobilized on the screen-printed graphene [40]. Then, utilizing cyclic voltammetry as an electrochemical technique, the immobilized DNA on a graphene system that has been exposed to a sample (that may or may not contain the EN2 biomarker), can give an electrochemical signal indicating whether the EN2 biomarker is present. An example of a sensor based on this system has a LOD of $38.5 \mathrm{nmol} \cdot \mathrm{L}^{-1}$ with a linear range of $35-185 \mathrm{nmol} \cdot \mathrm{L}^{-1}$. This sensor has a relatively high level of sensitivity considering it is based only on graphene [41].

Graphene nanosheets are used in such assemblies in conjunction with other materials as hybrids coupled to create nanosystems which exhibit enhanced sensitivity. One such system uses a composite of graphene nanosheets and polyaniline as a platform for PSA detection [42]. Polyaniline provides a conductive polymer to the nanostructure, and it has an acid/base doping response, which allows it to be used in biosensors [43]. The resulting sensors utilizing this systems has a LOD of $3.3 \times 10^{-17} \mathrm{~mol} \cdot \mathrm{L}^{-1}$ and a wide linear range of $1.0 \times 10^{-16}$ to $1.0 \times 10^{-8} \mathrm{~mol} \cdot \mathrm{L}^{-1}$, hence showing promise in applications for PSA sensors.

Other sensors recently reported incorporate poly-Llactide nanopolymers embedded with graphene oxide nanosystems that has been screen-printed onto a gold electrode [44]. The effect of the poly-L-lactide was to amplify the electrochemical signal once exposed to the PSA antigen, in an effort to increase sensitivity of the sensor. The resulting LOD was $1 \mathrm{ng} \cdot \mathrm{mL}^{-1}$ towards PSA, and although this does not perform as well compared to

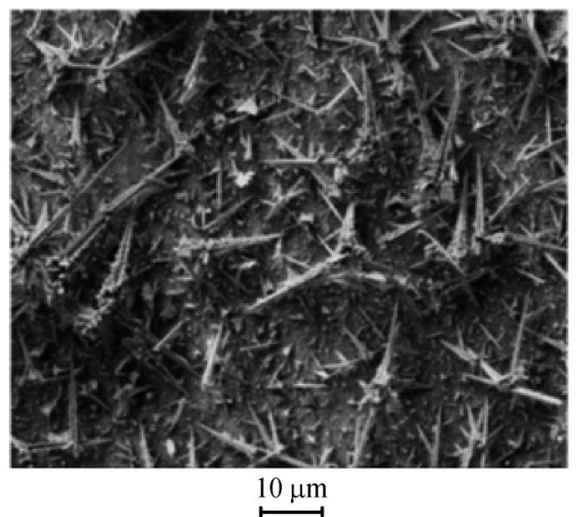

(a)

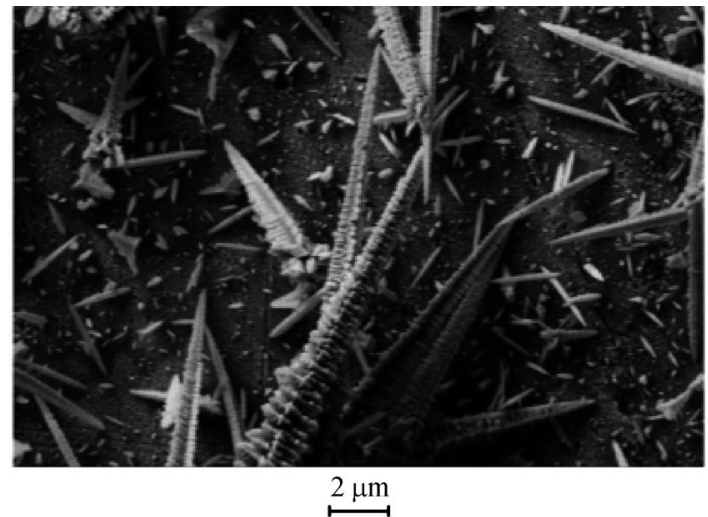

(b)

Fig. 5 Field emission scanning electron microscope (FESEM) images of nano-spindles (a) $10 \mu \mathrm{m}$ range, and (b) $1 \mu \mathrm{m}$ range. Adapted from Ref. [34] 
later discussed hybrid sensors which can display a LOD as low as $3 \mathrm{pg} \cdot \mathrm{mL}^{-1}$, this sensor is capable of dual detection of another biomarker. This novel design has the capability for detecting VEGF, as shown in Fig. 6, due to the use of a gold electrode, this just goes to prove that application of nanostructures to existing sensors can improve their application capability.

Further research into reduced graphene oxide nanosystems yields a sensor based on reduced graphene oxide functionalized with iron oxydroxide $(\mathrm{FeOOH})$, which is a signal-on photoelectrochemical immunosensor for detecting PSA [45]. Having $\mathrm{FeOOH}$ incorporated into the reduced graphene oxide allows the system to become photoactive, as the reduced graphene oxide acts as an electron mediator greatly facilitates the electron transfer from $\mathrm{FeOOH}$ to electrode under visible light [46]. Experimental results from this study show the sensor to be effective at detecting PSA in the range of $1 \mathrm{pg} \cdot \mathrm{mL}^{-1}$ to $100 \mathrm{ng} \cdot \mathrm{mL}^{-1}$, an excellent performance range indicating its prospect as a sensor for PSA.

\subsection{Gold-graphene hybrid materials for biosensing}

Alongside the main field of gold and graphene-based sensors, research into alternative designs, especially hybrid sensors, has become promising resulting in some very novel and effective examples. The idea behind a hybrid sensor is to gain the benefits of both sensor types and reducing any problems in a synergistic manner generating a more versatile sensor.

A study exhibiting a bare gold electrode against an impedimetric aptasensor electrode consisting of gold nanoparticles screen-printed onto a carbon ink electrode highlights the significant increase in sensitivity of hybrid sensors towards PSA. The bare gold electrode exhibited a
LOD of just $5 \mu \mathrm{g} \cdot \mathrm{mL}^{-1}$ compared to the hybrid sensor's LOD of $1.95 \mathrm{ng} \cdot \mathrm{mL}^{-1}$, indicating the superior detection ability of the composite aptasensor. The hybrid sensor also displayed high levels of selectivity, being able to distinguish PSA from three relevant competitor proteins found in this concentration range [47]. An example of such a sensor is a sensitive electrochemical aptasensor making use of a graphene electrode modified with gold nanoparticles described by graphitized mesoporous carbon nanoparticles. This sensor is based on a signal-off method, when the PSA aptamer captures the targeted PSA on the electrode there is a decrease in the differential pulse voltammetric signal of redox system indicating the presence of the antigen. This gives a way to quantitatively detect the PSA and in optimal conditions the PSA can be detected in the range of $0.25-200 \mathrm{ng} \cdot \mathrm{mL}^{-1}$, resulting in a highly sensitive sensor. This high sensitivity is due to the high amount of aptamer immobilized on the electrode surface, achieved by use of a biotin-streptavidin system, allowing not only a low detection limit but also a linear detection range up to $200 \mathrm{ng} \cdot \mathrm{mL}^{-1}$. The aptasensor was successfully added to serum samples with results being successfully obtained. This system could provide a promising platform for a future PSA detection methodology within a biosensor [48].

Other aptasensor has achieved an even higher sensitivity towards PSA, having a LOD of just $3 \mathrm{pg} \cdot \mathrm{mL}^{-1}$ and a wide linear range of $6 \mathrm{pg} \cdot \mathrm{mL}^{-1}$ to $30 \mathrm{ng} \cdot \mathrm{mL}^{-1}$. Such an improvement in sensitivity was achieved by fabricating a nanostructured electrode out of functionally reduced graphene oxide and applying gold nanoparticles to the electrode's surface before covalently immobilizing the monoclonal anti-PSA antibody to the sensor. Tested on human serum gave a recovery range of $97 \%$ to $110 \%$ with a relative standard deviation of $3.88 \%$, thus giving this
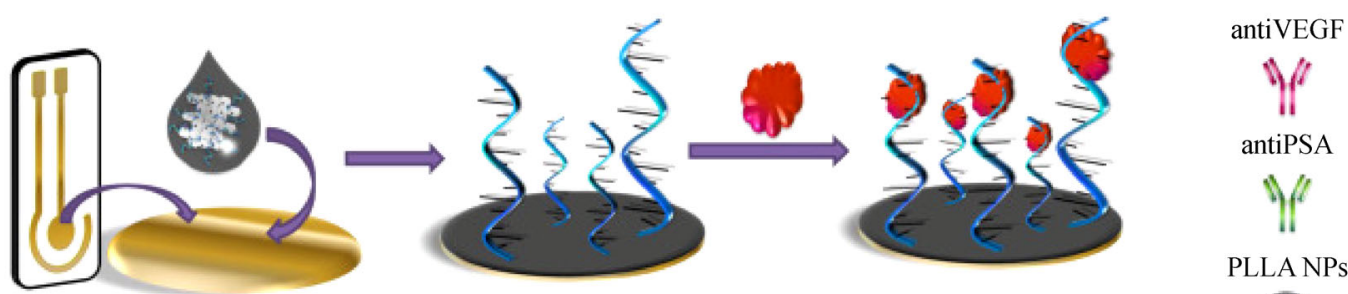

PLLA NPS
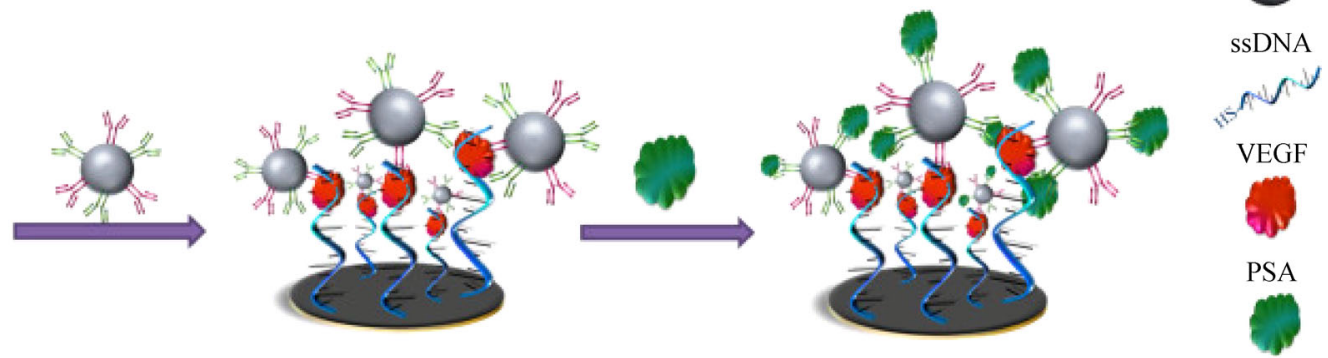

Fig. 6 Diagram showing dual capability of a sensor capable of detecting both vascular endothelial growth factor, of relevance in tumor microenvironment targeting (VEGF) and PSA. Adapted from Ref. [44] with permission from Elsevier, copyright (2017) 
sensor great potential in diagnostic applications [49].

Nanotechnology approaches applied to the design of bionanosensors for PSA detection also gave rise to three dimensional electrochemical immunosensor [50], taking the form of "crumpled" graphene balls decorated with gold nanoparticles. Figure 7 shows the structure of this novel structure imaged on the nanoscale $(200 \mathrm{~nm})$ using FESEM and TEM. This preparation was achieved by aerosol spray pyrolysis, and the unique sensor gives a LOD of $0.59 \mathrm{ng} \cdot \mathrm{mL}^{-1}$ with a linear range of $0 \mathrm{ng} \cdot \mathrm{mL}^{-1}$ to $10 \mathrm{ng} \cdot \mathrm{mL}^{-1}$. While other sensors are more sensitive than this novel example, this aptasensor represents the application of alternative nanomaterial designs in creating an effective PSA sensor.

3.4 Alternative sensor design for prostate cancer detection approaches

Although gold-graphene hybrid aptasensors are proving to be very effective in reducing LODs, research into sensors based on other nanotechnology has given some novel designs. Gold and graphene nano-structure systems have been more widely investigated, however other nanosystems based on other compounds or incorporating different elements could surpass the current sensors design.

Graphene oxide has demonstrated to be an effective synthetic scaffold layer for nanoparticle systems, as shown by a PSA immunosensor which comprises of a reduced graphene oxide electrode populated with silver nanoparticles [52]. The sensor displayed a wide linear response range of $1.0-1000 \mathrm{ng} \cdot \mathrm{mL}^{-1}$ and a low detection limit of $0.01 \mathrm{ng} \cdot \mathrm{mL}^{-1}$, this compared to the best performing graphene/gold aptasensor mentioned earlier [48] having a LOD of $0.25 \mathrm{ng} \cdot \mathrm{mL}^{-1}$. This could be due to the silver/ reduced graphene sensors exhibiting superior electrical conductivity as the silver particles were anchored to the reduced graphene oxide layer. This results in a very high sensitivity when measuring PSA which combined with the ability to modify the sensor for detecting other biomolecules provides the immunosensor with other potential applications in clinical diagnosis of prostate cancer [52].

The sandwich format approach shows promise in the biosensor design [33]. One novel silver nanoparticle-based sensor that utilizes the sandwich design comprised a first layer consisting of $\mathrm{Pd}-\mathrm{Ag}$ heterodimers loaded on aminofunctionalized multiwalled carbon nanotubes employed as labels of secondary antibodies. The other layer consisted of gold nanoparticles employed for the immobilization of primary antibodies [51]. Figure 8 shows the fabrication of the sensor and application in sensing PSA by monitoring the reduction of peroxide via cyclic voltammetry. The sandwich design gives the aptasensor a linear range of $0.1 \mathrm{pg} \cdot \mathrm{mL}^{-1}$ to $30 \mathrm{ng} \cdot \mathrm{mL}^{-1}$ and a LOD of $0.03 \mathrm{pg} \cdot \mathrm{mL}^{-1}$, such high sensitivity originated due to the "sandwich" like design compared to a direct method.

Other sensor based on reduced graphene oxide utilizes biomarker laden $\mathrm{Fe}_{3} \mathrm{O}_{4}$ nanoparticles loaded onto the graphene oxide nano-sheets [53]. This sensor shows exceptional sensitivity, with a LOD of just $15 \mathrm{fg} \cdot \mathrm{mL}^{-1}$ being one of the most sensitive sensors mentioned herein [49]. This sensitivity is attributed to the ability of the $\mathrm{Fe}_{3} \mathrm{O}_{4}$ /graphene oxide nano-particles to catalyse a hydrogen peroxide reduction to detect PSA, once the biomarker laden nanoparticles are captured by a second set of antibodies grafted onto a reduced graphene oxide electrode (Fig. 9). Additionally, the $\mathrm{Fe}_{3} \mathrm{O}_{4}$ nanoparticles serve the dual function of magnetic analyte isolation and labels for detection, meaning a low reagent cost. The reagent cost was only $\$ 0.85$ for a single 2-protein assay, which would translate in affordable deployment in population [53].
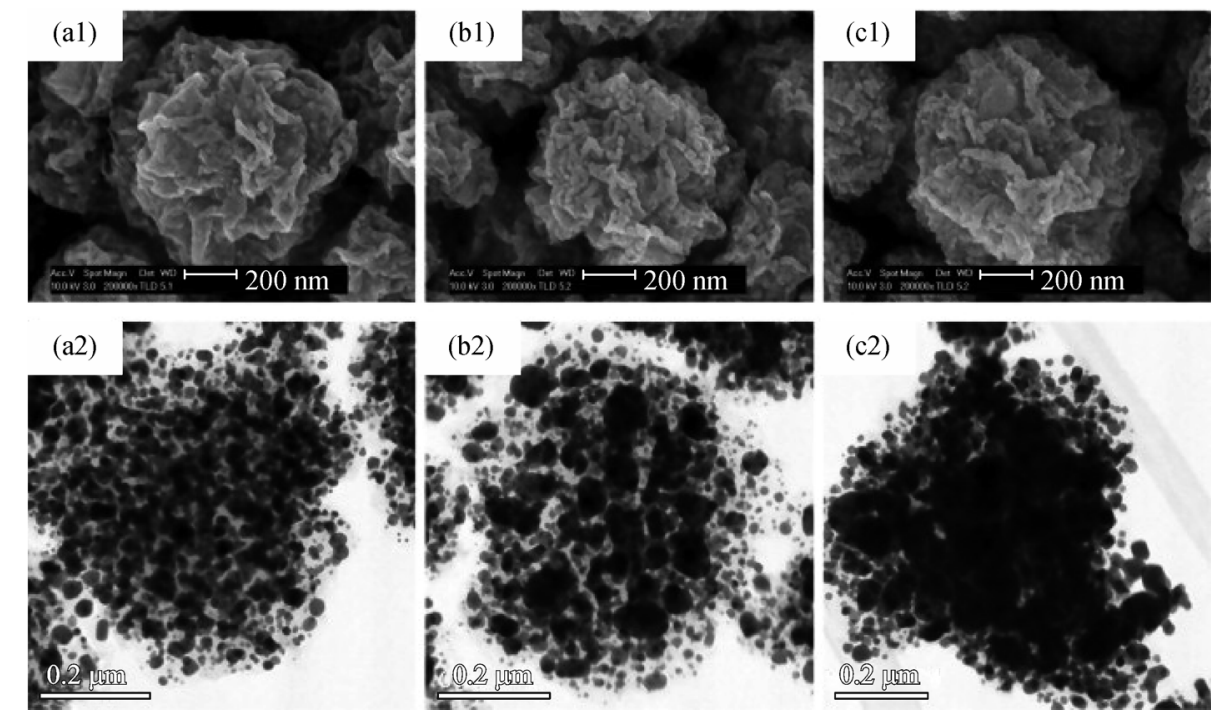

Fig. 7 FESEM (a1, b1, c1) and TEM (a2, b2, c2) images of the crumpled GR-Au composites. Adapted from Ref. [50] with permission from Elsevier, copyright (2015) 


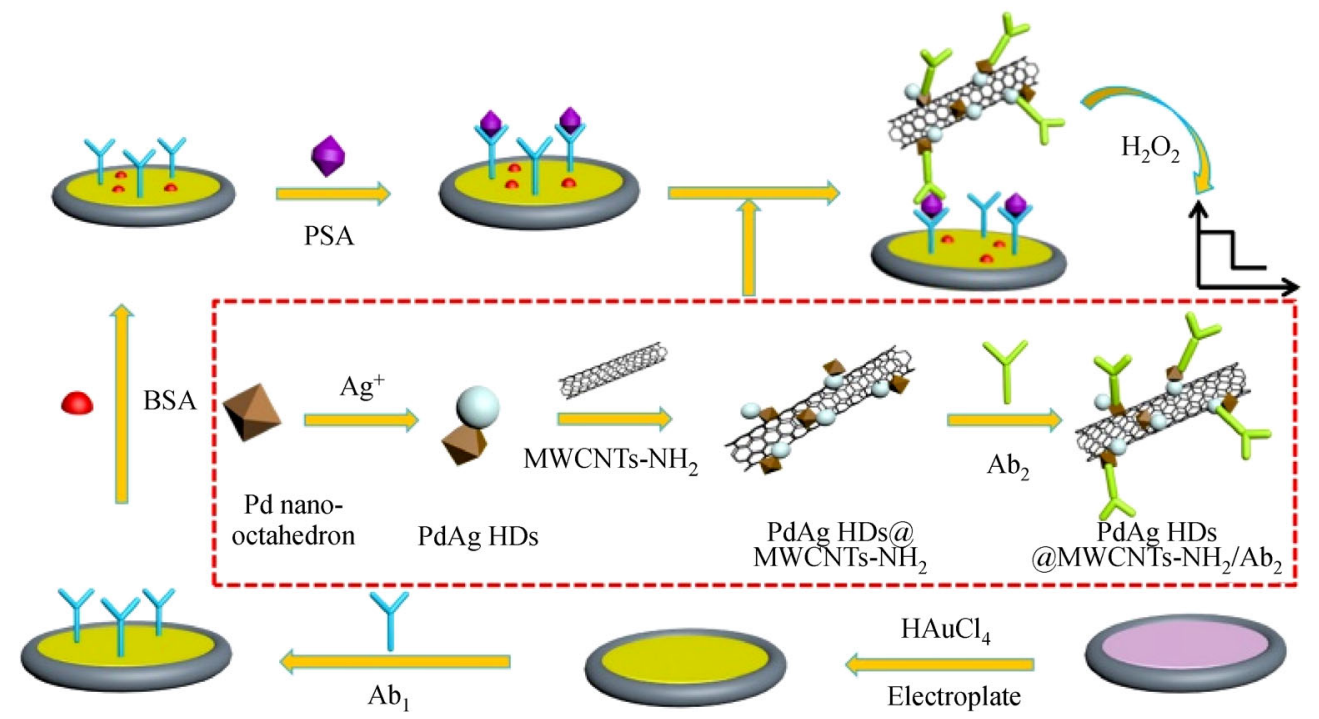

Fig. 8 Diagram showing immunosensor fabrication and reaction process when exposed to PSA. Adapted from Ref. [51] with permission from Elsevier, copyright (2016)

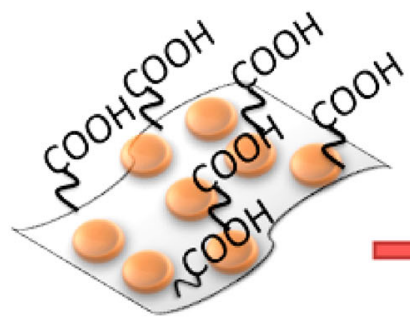

1. EDCNHSS

2. Antibody

3. $0.1 \%$ BSA

$\mathrm{Fe}_{3} \mathrm{O}_{4} @$ Graphene oxide
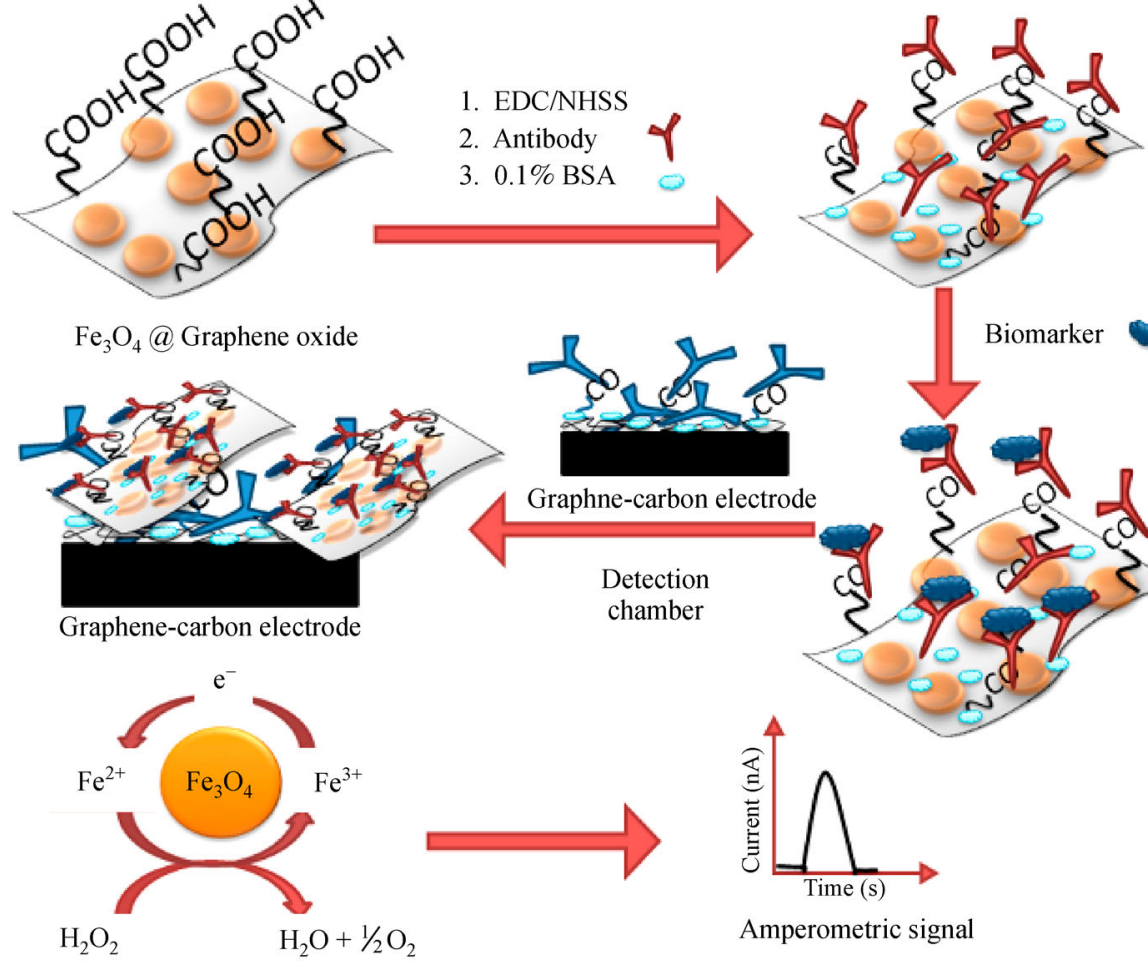

Biomarker

Amperometric signal

Fig. 9 PSA detection process with $\mathrm{Fe}_{3} \mathrm{O}_{4}$ /graphene oxide nanoparticles. Adapted from Ref. [53] with permission from Elsevier, copyright (2017)

An alternative sensor involved in the process of PSA detection by a sandwich-type aptasensor also relies on the catalytic $\mathrm{H}_{2} \mathrm{O}_{2}$ reduction giving a signal on electrochemical current (Fig. 10). This immunosensor shows a linear concentration range of $50 \mathrm{fg} \cdot \mathrm{mL}^{-1}$ to $40 \mathrm{ng} \cdot \mathrm{mL}^{-1}$, with a low detection limit of $16.6 \mathrm{fg} \cdot \mathrm{mL}^{-1}$, showing this design rivals other sensitive aptasensors [53].

\subsection{PSA detection by optical methods}

The use of optical methods of detection has led to the development of many biosensors due to the attractive advantages of this method such as direct, label free and real time measurements with possibilities of quantification [55]. 


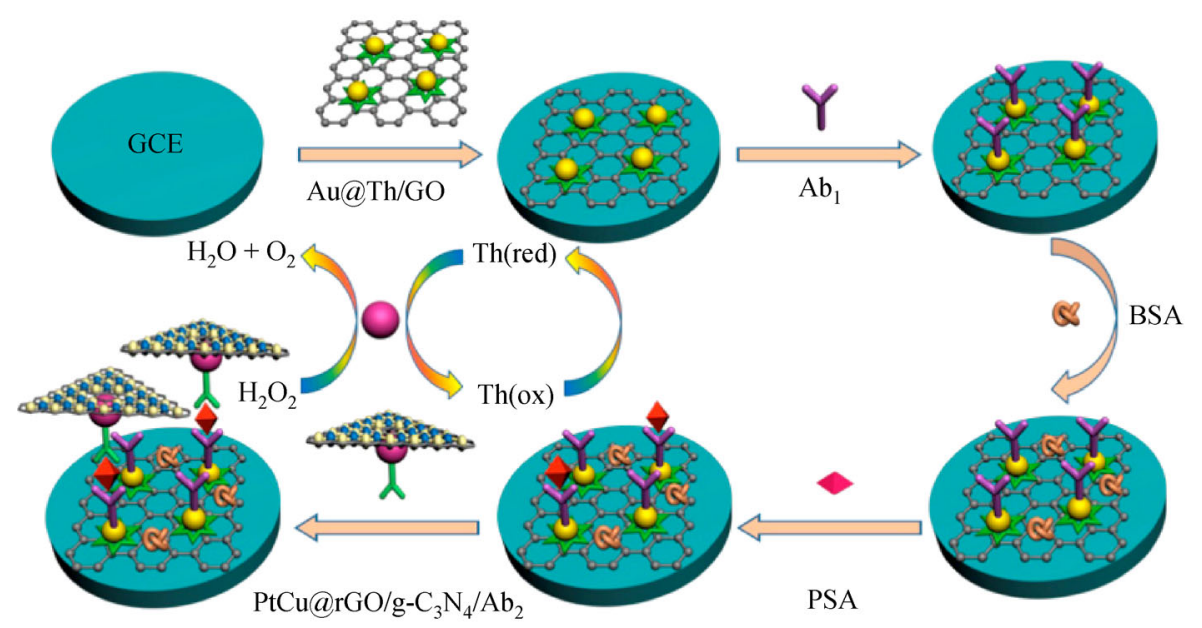

Fig. 10 Diagram showing process scheme of PSA detection for the novel sandwich-type aptasensor. Adapted from Ref. [54] with permission from Elsevier, copyright (2017)

There are several methods of optical PSA detection such as surface plasmon resonance (SPR), chemiluminescence, electrochemiluminescence or fluorescence. SPR is the method of choice for the study of many receptor - analyte interactions for the possibility of miniaturization, label free analysis, minimum amount of sample required or reusable sample chips. SPR generally involves the immobilization of a ligand in a metallic surface in which the analyte is introduced in a fluidic setup. The binding of the analyte produces surface plasmons that are detected by the change in intensity and refractive index of a polarized light source illuminated at the back of the detector in the interface of the metallic/glass surface. The main disadvantage of SPR biosensors might be the high detection limits so gold nanoparticles [56,57], silver nanoprisms [58] or nanocomposites $\left(\mathrm{MoS}_{2} \mathrm{QDs} @ \mathrm{~g}-\mathrm{C}_{3} \mathrm{~N}_{4} @ \mathrm{CS}-\mathrm{AuNPs}\right)$ [59] have been employed in order to amplify the obtained signals in the detection of PSA reaching limits of detection in the $\mathrm{ng} \cdot \mathrm{mL}^{-1}[56,57,59]$ and $\mathrm{fg} \cdot \mathrm{mL}^{-1}$ respectively [58].

Electroluminescence (ECL) makes use of electrochemical energy to allow a fluorophore to access an excited state which emits light in its decay. ECL presents a series of advantages that makes it highly attractive for the construction of biosensors such as the lack of a light source which eliminates the presence of a background, the low detection limits, stabilities of the systems used, the use of simple instrumentation or the possibility of signal enhancement by the use of nanoparticles. In addition, ECL is considered more selective than chemiluminescence (CL) as it can be controlled by the applied potential $[60,61]$. There are two commonly used ECL systems: one formed by luminol and hydrogen peroxide and the the other comprising $\mathrm{Ru}(\mathrm{bpy})^{3+}$ species and tripropylamine. In both cases, the fluorophore is oxidized electrochemically in the presence of the secondary reagent (Fig. 11) [61].

Nanomaterials have been applied to the detection of PSA in ECL setups circumventing the need of chemical systems due to the possibilities of nanoparticles to emit when excited with an electrical current and providing considerable enhancement of the signal and improvement of limits of detection. Flower-like nano $\mathrm{TiO}_{2}$ was applied as the luminescent material in a sandwich type immunoassay for the determination of PSA. The roughness of the $\mathrm{TiO}_{2}$ nanoparticles helped to increase the ECL intensity when compared to smooth NPs and allowed for the detection of PSA to the $\mathrm{pg} \cdot \mathrm{mL}^{-1}$ level in spiked human (a)<smiles>Nc1cccc(C(=O)[O-])c1C(=O)OCCCCO</smiles>

$+\mathrm{N}_{2}+2 \mathrm{H}_{2} \mathrm{O}+2 \mathrm{H}^{+}+h v\left(\lambda_{\max }=425 \mathrm{~nm}\right)$

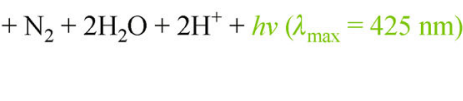

(b) $\mathrm{Ru}\left(\mathrm{bpy}_{3}\right)^{2+}+\mathrm{TPA}$

$\longrightarrow \mathrm{Ru}\left(\mathrm{bpy}_{3}\right)^{3+}+\mathrm{TPA}^{\cdot}$

$\longrightarrow \operatorname{Ru}\left(\mathrm{bpy}_{3}\right)^{2+}+h v\left(\lambda_{\max }=610 \mathrm{~nm}\right)$

Tripropylamine (TPA)

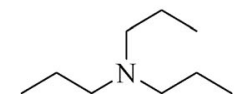

Fig. 11 Simplified ECL mechanism of (a) luminol and (b) $\mathrm{Ru}\left(\mathrm{bpy}_{3}\right)^{2+}$ 
serum samples [62]. In a similar fashion, a label free method was developed based on silver nanoparticles doped with $\mathrm{Pb}$ (II) incorporated into a metal cyclodextrin organic framework with comparable limits of detection [63]. Quantum dots showed to be able to undergo emission under ECL settings and in consequence they have been applied to the construction of ECL PSA biosensors $[64,65]$.

The introduction of carbon nanomaterials, particularly graphene, into biosensor research thanks to the advantageous properties has also been applied to ECL devices in combination with other nanomaterials. An "on-off" switchable effect was achieved from the adsorption of gold nanoparticles - CdS "flower" like nanohybrids functionalized with PSA aptamers onto graphene sheets. The aptasensors switched to the "on" state in the presence of PSA showing good limits of detection and potential for the detection of other targets of interest [66]. Other nanohybrid structure containing graphene was formed by the combination of titanium dioxide nanotubes infilled with graphene quantum dots. The authors reported a greater analytical performance of the nanohybrids compared to the nanomaterials alone for the detection of PSA in the sandwich immunoassay setup [67]. ECL aptasensors based on the luminol ECL effect incorporated nanomaterials as a way of enhancing the output signal. Graphene oxide-gold nanorod-glucose oxidase - DNA nanocomposites were reported to show signal amplification over a wide linear range for the detection of PSA [28].

The production of a fluorescent signal has also been employed to develop PSA biosensors thanks to the high sensitivity of fluorescent techniques and the development of selective fluorescent tags and nanomaterials. Systems based on gold and silica nanoparticles have been widely explored due to ease in functionalization and low toxicity of these materials.

The self-assembly of gold-upconversion-nanoparticle pyramids with aptamers was studied for the detection of PSA and thrombin by SERS and fluorescent detection [69]. The system provided ultrasensitive detection of the targets and in the case of fluorescent detection a fluorescent increase was observed in the presence of the target molecule.

Silica nanospheres have been applied to aggregationinduced emission nanosystems which resulted in a "turn- on" effect after PSA recognition by the aptamer functionalized surface [68]. The sensor demonstrated a LOD of $0.5 \mathrm{ng} \cdot \mathrm{mL}^{-1}$ which is similar to gold nanoparticle based sensors. Once the binding of the PSA aptamer to the target PSA occurs, a rigid aptamer conformation is induced, resulting in the release of the PSA aptamer away from the surface of silica nanospheres. This made the aggregationinduced emission molecules incorporated into the nanospheres aggregate on the spheres' surface, giving a high fluorescence emission (Fig. 12). This versatile design is simple and highly selective towards PSA, giving it the potential to be used in clinical or diagnostic applications.

Other "off-on" PSA biosensors rely on a $\mathrm{Fe}_{3} \mathrm{O}_{4} @ \mathrm{SiO}_{2}$ $\mathrm{Au}$ nanocomposite with a functionalized surface of fluorescent 5-FAM peptides (Fig. 13) [70]. The nanocomposite was formed sequentially by encapsulating the nanocubes of $\mathrm{Fe}_{3} \mathrm{O}_{4}$ into a silica particle followed by attachment of gold nanoparticles and self-assembly of the 5-FAM peptides, quenched in the presence of the composite. The "off-on" effect was achieved when the peptidic layer was selectively cleaved in the presence of PSA and the fluorescence was recovered.

The magnetic nature of the nanocomposite allowed for an easy separation upon recognition of PSA to avoid any undesired background signal. Silver nanoparticles have also been encapsulated within silica layers to produce a biosensing nanocomposite $\left(\mathrm{Ag} @ \mathrm{SiO}_{2} @ \mathrm{SiO}_{2}-\left[\mathrm{Ru}(\mathrm{bpy})_{3}\right]^{2+}\right.$ towards PSA [71]. In this case, an organic fluorophore has been employed, taking advantage of the luminescent enhancement provided by the interaction of the $\left[\mathrm{Ru}(\mathrm{bpy})_{3}\right]^{2+}$ and the silver cores, the PSA selectivity was achieved thanks to a PSA antibody and magnetic separation was possible after analysis. A strategy in a similar fashion but relying on the quenching of up-conversion nanoparticles functionalized with anti-PSA antibodies in a sandwich type immunoassay has been reported. The single particle counting in a microscope setup allowed for PSA limits of detection as low as $1 \mathrm{pmol} \cdot \mathrm{L}^{-1}[72]$.

The use of quantum dots (QDs) has also proved popular in the construction of PSA sensors, especially in setups with other fluorophores by measuring of Förster resonance energy transfer (FRET) interactions [73]. QDs present many advantages beyond their tunable absorptions/emissions for the preparation of this kind of assays such as low LOD, multiplexing capability or ability to use body fluids

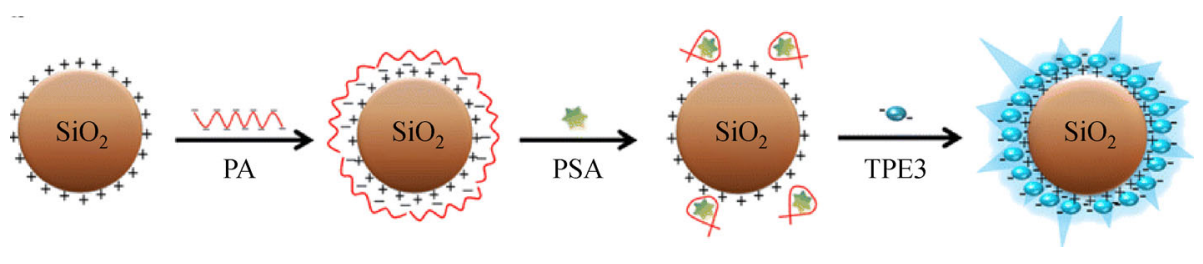

Fig. 12 Diagram showing process scheme of PSA detection giving a florescence emission. Adapted from Ref. [68] with permission from Springer Nature, copyright (2017) 


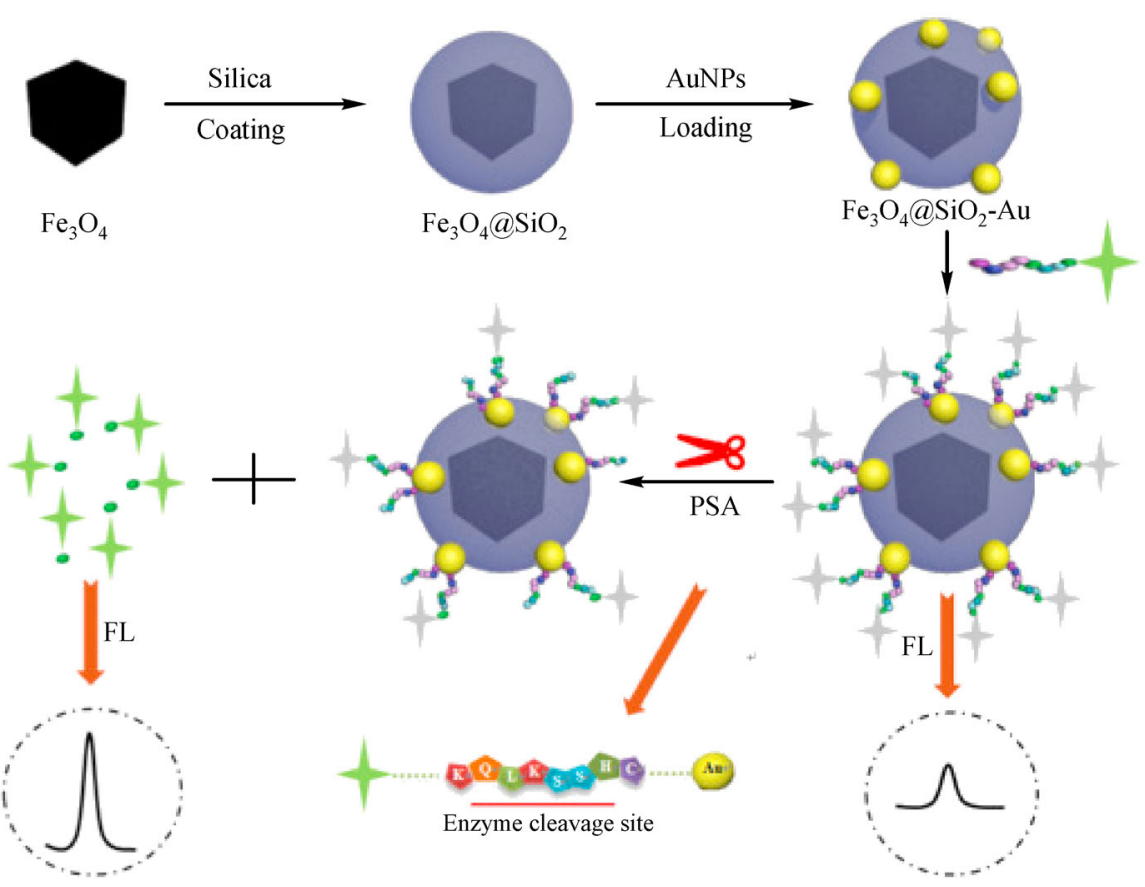

Fig. 13 Diagram of peptide/MNCPs sensor for the fluorescent detection of PSA. Adapted from Ref. [70] with permission from Elsevier, copyright (2018)

without extensive sample treatment [74]. Quantum dots have been labelled with antibodies in sandwich immunoassays and although detection has been reported by mass spectrometry [75] or photoelectrochemically [76] the most widespread detection method is fluorescence or FRET so similar approaches have been applied to quantum dots involving aptamers and antibodies for fluorescent PSA detection. Sensitivity enhancements have been observed by increasing the conjugation of antibodies to the QDs [77] or by preparing compact conjugates with fragmented antibodies (total hydrodynamic diameter of ca. $13 \mathrm{~nm}$ ) [78], achieving LODs below $1 \mathrm{ng} \cdot \mathrm{mL}^{-1}$ in the latter. The functionalization with dendrimers allows for a higher loading ability and the immune-complex formed with PSA could be analyzed by FRET [79], functionalization with zwitterionic penicillamine allowed for aqueous phase transfer [78], or the grafting onto a surface allowed for sensing devices aimed to point-of-care purposes towards $\mathrm{PCa}$ sensing investigations [80,81].

Alternative use of $2 \mathrm{D}$ materials such as graphene, graphene oxide, or $\mathrm{MoS}_{2}$ have led to increased promise for fluorescent detection of PSA, generally through a dye quenching process or "on-off" process. $\mathrm{MoS}_{2}$ and other Mo nanosheets $\left(\mathrm{MoO}_{3}, \mathrm{MoS}_{2}, \mathrm{MoSe}_{2}\right)$ can be employed in this way experiencing a "turn-on" process on binding of the aptamer to the PSA target $[19,82]$. Graphene oxide composites such as graphene oxide quantum dots@silver nanocrystals or QD-aptamer GO nanocomposites were employed in the same way for detection of PSA $[83,84]$.

\section{Overview and conclusions}

Overall, gold nanoparticles and graphene nanostructures represent the main components of nanotechnology towards immunosensors for PSA. The introduction of both of these nanomaterials or hybrid systems comprising a combination of both allowed for the increase in versatility and sensitivity when compared to traditional analytical methods, such as ELISA.

The current limit of detection that a gold sensor can display a LOD are of ca. $10 \mathrm{pg} \cdot \mathrm{mL}^{-1}$ while a hybrid system combining gold nanoparticles and graphene nanosheets manage to reach a LOD of $0.59 \mathrm{ng} \cdot \mathrm{mL}^{-1}$. While gold and graphene are effective materials for fabricating nanosystems for biosensing of PSA, the best sensitivity discovered by this report was exhibited by a sandwich-type electrochemical immunosensor based on gold loaded on thionine functionalized graphene oxide and reduced graphene oxide/graphitic carbon nitride loaded with $\mathrm{PtCu}$ bimetallic hybrid nanoparticles. This displayed a LOD of just $16.6 \mathrm{fg} \cdot \mathrm{mL}^{-1}$, by far the most sensitive sensor in this report, and as such, designs similar to this should be the focus of continued research.

Without question, immunosensors for detecting PSA based on nanotechnology outperform the current clinical tests for PSA. Most notably, the aptasensors with nanostructures display a vast improvement in sensitivity and specificity towards PSA when compared to conventional test methods. These sensors eliminate the problems 
faced by current tests, they are non-invasive, rapid and the tests are simple enough to be performed by untrained personnel in community settings and 'GP' centers. Additionally, once the designs of such sensors are optimized and validated, they appear to be rather simple to fabricate on a commercial scale. Hence use of such technology in clinical environments will not only smoothen out the process of diagnosing prostate cancer, but also make the whole process likely to benefit of patient acceptability. Prostate cancer has been the focus of many research groups from a variety of disciplines, and now with the application of nanotechnology into the sensors' design and fabrication, scientists are a step closer to detecting earlier this non-communicable disease. The applications and mechanisms discovered by applying biosensing principles to PSA detection will be able to be applied to other diseases, including non-communicable diseases, and hence combat other diseases with significant social and economic benefit.

The difference in sensitivity is not necessarily the deciding factor when it comes to selecting a design best suited for a clinical application for detecting PSA. Problems facing conventional tests are a severe lack in specificity and reproducibility, leading to false results. Reproducible and reliable results are what is required for the clinical diagnostic application of a sensor, and this concept is one of the reasons nanotechnology has been researched for this application. The goal of being able to monitor and detect prostate cancer more efficiently has been considered for future work, the end goal ultimately being the fabrication of a sensor, which can be deployed into the general public. Giving the general population the ability to monitor levels of PSA could allow for the process of detecting and treating prostate cancer to become more effective. This could increase the quality of prognoses of prostate cancer diagnoses, and in turn improve the quality of the life of all effected individuals.

\begin{abstract}
Acknowledgements The authors would like to thank the ERC Consolidator grant scheme (O2SENSE) for funding PROSENSE network for funding and Dr. Pedro Estrela for a collaboration and supportive discussions in this field.

Open Access This article is licensed under a Creative Commons Attribution 4.0 International License, which permits use, sharing, adaptation, distribution and reproduction in any medium or format, as long as you give appropriate credit to the original author(s) and the source, provide a link to the Creative Commons licence, and indicate if changes were made. The images or other third party material in this article are included in the article's Creative Commons licence, unless indicated otherwise in a credit line to the material. If material is not included in the article's Creative Commons licence and your intended use is not permitted by statutory regulation or exceeds the permitted use, you will need to obtain permission directly from the copyright holder. To view a copy of this licence, visit http://creativecommons.org/licenses/by/4.0/.
\end{abstract}

\section{References}

1. Ferlay J, Colombet M, Soerjomataram I, Dyba T, Randi G, Bettio
M, Gavin A, Visser O, Bray F. Cancer incidence and mortality patterns in Europe: Estimates for 40 countries and 25 major cancers in 2018. European Journal of Cancer, 2018, 103: 356-387

2. Luengo-Fernandez R, Leal J, Gray A, Sullivan R. Economic burden of cancer across the European Union: A population-based cost analysis. Lancet. Oncology, 2013, 14(12): 1165-1174

3. Siegel R L, Miller K D, Jemal A. Cancer statistics, 2018. CA: A Cancer Journal for Clinicians, 2018, 68(1): 7-30

4. Ge H, Riss P J, Mirabello V, Calatayud D G, Flower S E, Arrowsmith R L, Fryer T D, Hong Y, Sawiak S, Jacobs R M J, et al. Behavior of supramolecular assemblies of radiometal-filled and fluorescent carbon nanocapsules in vitro and in vivo. Chem, 2017, 3 (3): $437-460$

5. Kumar C S S R, Mohammad F. Magnetic nanomaterials for hyperthermia-based therapy and controlled drug delivery. Advanced Drug Delivery Reviews, 2011, 63(9): 789-808

6. Heidenreich A, Bastian P J, Bellmunt J, Bolla M, Joniau S, van der Kwast T, Mason M, Matveev V, Wiegel T, Zattoni F, Mottet N. EAU guidelines on prostate cancer. Part 1: Screening, diagnosis, and local treatment with curative intent-update 2013. European Urology, 2014, 65(1): 124-137

7. Force U S P S T. Screening for prostate cancer: US preventive services task force recommendation statement. Journal of the American Medical Association, 2018, 319(18): 1901-1913

8. Yao J, Wang Y, Dai Y, Liu C C. Bioconjugated, single-use biosensor for the detection of biomarkers of prostate cancer. ACS Omega, 2018, 3(6): 6411-6418

9. Rigau M, Ortega I, Mir M C, Ballesteros C, Garcia M, Llauradó M, Colás E, Pedrola N, Montes M, Sequeiros T, et al. A three-gene panel on urine increases PSA specificity in the detection of prostate cancer. Prostate, 2011, 71(16): 1736-1745

10. Salami S S, Schmidt F, Laxman B, Regan M M, Rickman D S, Scherr D, Bueti G, Siddiqui J, Tomlins S A, Wei J T, et al. Combining urinary detection of TMPRSS2:ERG and PCA3 with serum PSA to predict diagnosis of prostate cancer. Urologic Oncology: Seminars and Original Investigations, 2013, 31(5): 566-571

11. Lundwall $\AA$, Lilja H. Molecular cloning of human prostate specific antigen cDNA. FEBS Letters, 1987, 214(2): 317-322

12. Stura E A, Muller B H, Bossus M, Michel S, Jolivet-Reynaud C, Ducancel F. Crystal structure of human prostate-specific antigen in a sandwich antibody complex. Journal of Molecular Biology, 2011, 414(4): 530-544

13. Cheng Z, Choi N, Wang R, Lee S, Moon K C, Yoon S Y, Chen L, Choo J. Simultaneous detection of dual prostate specific antigens using surface-enhanced Raman scattering-based immunoassay for accurate diagnosis of prostate cancer. ACS Nano, 2017, 11(5): 4926-4933

14. Voller A, Bartlett A, Bidwell D E. Enzyme immunoassays with special reference to ELISA techniques. Journal of Clinical Pathology, 1978, 31(6): 507-520

15. Acevedo B, Perera Y, Ruiz M, Rojas G, Benítez J, Ayala M, Gavilondo J. Development and validation of a quantitative ELISA for the measurement of PSA concentration. Clinica Chimica Acta, 2002, 317(1): 55-63

16. Luderer A A, Chen Y T, Soriano T F, Kramp W J, Carlson G, Cuny 
C, Sharp T, Smith W, Petteway J, Brawer M K, et al. Measurement of the proportion of free to total prostate-specific antigen improves diagnostic performance of prostate-specific antigen in the diagnostic gray zone of total prostate-specific antigen. Urology, 1995, 46(2): 187-194

17. Chen Z, Lei Y, Chen X, Wang Z, Liu J. An aptamer based resonance light scattering assay of prostate specific antigen. Biosensors \& Bioelectronics, 2012, 36(1): 35-40

18. Sarkar P, Pal P S, Ghosh D, Setford S J, Tothill I E. Amperometric biosensors for detection of the prostate cancer marker (PSA). International Journal of Pharmaceutics, 2002, 238(1): 1-9

19. Dhenadhayalan N, Yadav K, Sriram M I, Lee H L, Lin K C. Ultrasensitive DNA sensing of a prostate-specific antigen based on $2 \mathrm{D}$ nanosheets in live cells. Nanoscale, 2017, 9(33): 12087-12095

20. Jolly P, Tamboli V, Harniman R L, Estrela P, Allender C J, Bowen J L. Aptamer-MIP hybrid receptor for highly sensitive electrochemical detection of prostate specific antigen. Biosensors \& Bioelectronics, 2016, 75: 188-195

21. Choi J H, Kim H S, Choi J W, Hong J W, Kim Y K, Oh B K. A novel $\mathrm{Au}$-nanoparticle biosensor for the rapid and simple detection of PSA using a sequence-specific peptide cleavage reaction. Biosensors \& Bioelectronics, 2013, 49: 415-419

22. Chikkaveeraiah B V, Bhirde A A, Morgan N Y, Eden H S, Chen X. Electrochemical Immunosensors for Detection of Cancer Protein Biomarkers. ACS Nano, 2012, 6(8): 6546-6561

23. Souada M, Piro B, Reisberg S, Anquetin G, Noël V, Pham M C. Label-free electrochemical detection of prostate-specific antigen based on nucleic acid aptamer. Biosensors \& Bioelectronics, 2015, 68: $49-54$

24. Damborska D, Bertok T, Dosekova E, Holazova A, Lorencova L, Kasak P, Tkac J. Nanomaterial-based biosensors for detection of prostate specific antigen. Mikrochimica Acta, 2017, 184(9): 30493067

25. Pfister C, Basuyau J P. Current usefulness of free/total PSA ratio in the diagnosis of prostate cancer at an early stage. World Journal of Urology, 2005, 23(4): 236-242

26. Dong Y X, Cao J T, Liu Y M, Ma S H. A novel immunosensing platform for highly sensitive prostate specific antigen detection based on dual-quenching of photocurrent from CdSe sensitized $\mathrm{TiO}_{2}$ electrode by gold nanoparticles decorated polydopamine nanospheres. Biosensors \& Bioelectronics, 2017, 91: 246-252

27. Wang Y, Li Z, Hu D, Lin C T, Li J, Lin Y. Aptamer/graphene oxide nanocomplex for in situ molecular probing in living cells. Journal of the American Chemical Society, 2010, 132(27): 9274-9276

28. Cao J T, Yang J J, Zhao L Z, Wang Y L, Wang H, Liu Y M, Ma S H. Graphene oxide@gold nanorods-based multiple-assisted electrochemiluminescence signal amplification strategy for sensitive detection of prostate specific antigen. Biosensors \& Bioelectronics, 2018, 99: 92-98

29. Wang X, Xu R, Sun X, Wang Y, Ren X, Du B, Wu D, Wei Q. Using reduced graphene oxide-Ca:CdSe nanocomposite to enhance photoelectrochemical activity of gold nanoparticles functionalized tungsten oxide for highly sensitive prostate specific antigen detection. Biosensors \& Bioelectronics, 2017, 96: 239-245

30. Yang Z, Kasprzyk-Hordern B, Goggins S, Frost C G, Estrela P. A novel immobilization strategy for electrochemical detection of cancer biomarkers: DNA-directed immobilization of aptamer sensors for sensitive detection of prostate specific antigens. Analyst (London), 2015, 140(8): 2628-2633

31. Wu M S, Chen R N, Xiao Y, Lv Z X. Novel "signal-on" electrochemiluminescence biosensor for the detection of PSA based on resonance energy transfer. Talanta, 2016, 161: 271-277

32. Jolly P, Zhurauski P, Hammond J L, Miodek A, Liébana S, Bertok T, Tkáč J, Estrela P. Self-assembled gold nanoparticles for impedimetric and amperometric detection of a prostate cancer biomarker. Sensors and Actuators. B, Chemical, 2017, 251: 637643

33. Kavosi B, Salimi A, Hallaj R, Moradi F. Ultrasensitive electrochemical immunosensor for PSA biomarker detection in prostate cancer cells using gold nanoparticles/PAMAM dendrimer loaded with enzyme linked aptamer as integrated triple signal amplification strategy. Biosensors \& Bioelectronics, 2015, 74: 915-923

34. Sattarahmady N, Rahi A, Heli H. A signal-on built in-marker electrochemical aptasensor for human prostate-specific antigen based on a hairbrush-like gold nanostructure. Scientific Reports, 2017, 7(1): 11238

35. Geim A K, Novoselov K S. The rise of graphene. Nature Materials, 2007, 6(3): 183-191

36. poor N Z M, Baniasadi L, Omidi M, Amoabediny G, Yazdian F, Attar H, Heydarzadeh A, Zarami A S H, Sheikhha M H. An inhibitory enzyme electrode for hydrogen sulfide detection. Enzyme and Microbial Technology, 2014, 63: 7-12

37. Andronescu C, Schuhmann W. Graphene-based field effect transistors as biosensors. Current Opinion in Electrochemistry, 2017, 3(1): 11-17

38. Shang N G, Papakonstantinou P, McMullan M, Chu M, Stamboulis A, Potenza A, Dhesi S S, Marchetto H. Catalyst-free efficient growth, orientation and biosensing properties of multilayer graphene nanoflake films with sharp edge planes. Advanced Functional Materials, 2008, 18(21): 3506-3514

39. Zhang J J, Gu M M, Zheng T T, Zhu J J. Synthesis of gelatinstabilized gold nanoparticles and assembly of carboxylic singlewalled carbon nanotubes/au composites for cytosensing and drug uptake. Analytical Chemistry, 2009, 81(16): 6641-6648

40. McGrath S E, Michael A, Pandha H, Morgan R. Engrailed homeobox transcription factors as potential markers and targets in cancer. FEBS Letters, 2013, 587(6): 549-554

41. Settu K, Liu J T, Chen C J, Tsai J Z. Development of carbongraphene-based aptamer biosensor for EN2 protein detection. Analytical Biochemistry, 2017, 534: 99-107

42. Tezerjani M D, Benvidi A, Rezaeinasab M, Jahanbani S, Moshtaghioun S M, Youssefi M, Zarrini K. An impedimeric biosensor based on a composite of graphene nanosheets and polyaniline as a suitable platform for prostate cancer sensing. Analytical Methods, 2016, 8(41): 7507-7515

43. Gao X Z, Liu H J, Cheng F, Chen Y. Thermoresponsive polyaniline nanoparticles: Preparation, characterization, and their potential application in waterborne anticorrosion coatings. Chemical Engineering Journal, 2016, 283: 682-691

44. Pan L H, Kuo S H, Lin T Y, Lin C W, Fang P Y, Yang H W. An electrochemical biosensor to simultaneously detect VEGF and PSA for early prostate cancer diagnosis based on graphene oxide/ssDNA/ 
PLLA nanoparticles. Biosensors \& Bioelectronics, 2017, 89: 598605

45. Zhou Q, Lin Y, Shu J, Zhang K, Yu Z, Tang D. Reduced graphene oxide-functionalized $\mathrm{FeOOH}$ for signal-on photoelectrochemical sensing of prostate-specific antigen with bioresponsive controlled release system. Biosensors \& Bioelectronics, 2017, 98: 15-21

46. Padhi D K, Parida K. Facile fabrication of $\alpha-\mathrm{FeOOH}$ nanorod/RGO composite: A robust photocatalyst for reduction of $\mathrm{Cr}(\mathrm{VI})$ under visible light irradiation. Journal of Materials Chemistry. A, Materials for Energy and Sustainability, 2014, 2(26): 10300-10312

47. Do T T N, Van Phi T, Nguy T P, Wagner P, Eersels K, Vestergaard M C, Truong L T N. Anisotropic in situ-coated AuNPs on screenprinted carbon surface for enhanced prostate-specific antigen impedimetric aptasensor. Journal of Electronic Materials, 2017, 46 (6): 3542-3552

48. Liu B, Lu L S, Hua E, Jiang S T, Xie G M. Detection of the human prostate-specific antigen using an aptasensor with gold nanoparticles encapsulated by graphitized mesoporous carbon. Mikrochimica Acta, 2012, 178(1-2): 163-170

49. Barman S C, Hossain M F, Park J Y. Gold nanoparticles assembled chemically functionalized reduced graphene oxide supported electrochemical immunosensor for ultra-sensitive prostate cancer detection. Journal of the Electrochemical Society, 2017, 164(6): B234-B239

50. Jang H D, Kim S K, Chang H, Choi J W. 3D label-free prostate specific antigen (PSA) immunosensor based on graphene-gold composites. Biosensors \& Bioelectronics, 2015, 63: 546-551

51. Liu L, Li Y, Tian L, Wei Q, Cao W. Ultrasensitive sandwich-type prostate specific antigen immunosensor based on Ag overgrowth in Pd nano-octahedrons heterodimers decorated on amino functionalized multiwalled carbon nanotubes. Sensors and Actuators. B, Chemical, 2016, 237: 733-739

52. Han L, Liu C M, Dong S L, Du C X, Zhang X Y, Li L H, Wei Y. Enhanced conductivity of $\mathrm{rGO} / \mathrm{Ag}$ NPs composites for electrochemical immunoassay of prostate-specific antigen. Biosensors \& Bioelectronics, 2017, 87: 466-472

53. Sharafeldin M, Bishop G W, Bhakta S, El-Sawy A, Suib S L, Rusling $\mathrm{J} \mathrm{F} . \mathrm{Fe}_{3} \mathrm{O}_{4}$ nanoparticles on graphene oxide sheets for isolation and ultrasensitive amperometric detection of cancer biomarker proteins. Biosensors \& Bioelectronics, 2017, 91: 359366

54. Feng J, Li Y, Li M, Li F, Han J, Dong Y, Chen Z, Wang P, Liu H, Wei Q. A novel sandwich-type electrochemical immunosensor for PSA detection based on $\mathrm{PtCu}$ bimetallic hybrid (2D/2D) rGO/g$\mathrm{C}_{3} \mathrm{~N}_{4}$. Biosensors \& Bioelectronics, 2017, 91: 441-448

55. Damborský P, Švitel J, Katrlík J. Optical biosensors. Essays in Biochemistry, 2016, 60(1): 91-100

56. Jiang Z, Qin Y, Peng Z, Chen S, Chen S, Deng C, Xiang J. The simultaneous detection of free and total prostate antigen in serum samples with high sensitivity and specificity by using the dualchannel surface plasmon resonance. Biosensors \& Bioelectronics, 2014, 62: 268-273

57. Zhang B, Liu B, Chen G, Tang D. Competitive-type displacement reaction for direct potentiometric detection of low-abundance protein. Biosensors \& Bioelectronics, 2014, 53: 465-471

58. Liang J, Yao C, Li X, Wu Z, Huang C, Fu Q, Lan C, Cao D, Tang Y.
Silver nanoprism etching-based plasmonic ELISA for the high sensitive detection of prostate-specific antigen. Biosensors \& Bioelectronics, 2015, 69: 128-134

59. Duan F, Zhang S, Yang L, Zhang Z, He L, Wang M. Bifunctional aptasensor based on novel two-dimensional nanocomposite of $\mathrm{MoS}_{2}$ quantum dots and $\mathrm{g}-\mathrm{C}_{3} \mathrm{~N}_{4}$ nanosheets decorated with chitosanstabilized Au nanoparticles for selectively detecting prostate specific antigen. Analytica Chimica Acta, 2018, 1036: 121-132

60. Parveen S, Aslam M S, Hu L, Xu G. Electrogenerated Chemiluminescence: Protocols and Applications. Dordrecht: Springer, 2013, $1-152$

61. Miao W. Electrogenerated chemiluminescence and its biorelated applications. Chemical Reviews, 2008, 108(7): 2506-2553

62. Deng W, Chu C, Ge S, Yu J, Yan M, Song X. Electrochemiluminescence PSA assay using an ITO electrode modified with gold and palladium, and flower-like titanium dioxide microparticles as ECL labels. Mikrochimica Acta, 2015, 182(5): 1009-1016

63. Ma H, Li X, Yan T, Li Y, Zhang Y, Wu D, Wei Q, Du B. Electrochemiluminescent immunosensing of prostate-specific antigen based on silver nanoparticles-doped $\mathrm{Pb}$ (II) metal-organic framework. Biosensors \& Bioelectronics, 2016, 79: 379-385

64. Shao K, Wang B, Nie A, Ye S, Ma J, Li Z, Lv Z, Han H. Targettriggered signal-on ratiometric electrochemiluminescence sensing of PSA based on MOF/Au/G-quadruplex. Biosensors \& Bioelectronics, 2018, 118: 160-166

65. Zhu W, Saddam Khan M, Cao W, Sun X, Ma H, Zhang Y, Wei Q. $\mathrm{Ni}(\mathrm{OH})_{2} / \mathrm{NGQDs}$-based electrochemiluminescence immunosensor for prostate specific antigen detection by coupling resonance energy transfer with $\mathrm{Fe}_{3} \mathrm{O}_{4} @ \mathrm{MnO}_{2}$ composites. Biosensors \& Bioelectronics, 2018, 99: 346-352

66. Yang J J, Cao J T, Wang H, Liu Y M, Ren S W. Ferrocene-graphene sheets for high-efficiency quenching of electrochemiluminescence from $\mathrm{Au}$ nanoparticles functionalized cadmium sulfide flower-like three dimensional assemblies and sensitive detection of prostate specific antigen. Talanta, 2017, 167: 325-332

67. Tian C, Wang L, Luan F, Zhuang X. An electrochemiluminescence sensor for the detection of prostate protein antigen based on the graphene quantum dots infilled $\mathrm{TiO}_{2}$ nanotube arrays. Talanta, 2019, 191: $103-108$

68. Kong R M, Zhang X, Ding L, Yang D, Qu F. Label-free fluorescence turn-on aptasensor for prostate-specific antigen sensing based on aggregation-induced emission-silica nanospheres. Analytical and Bioanalytical Chemistry, 2017, 409(24): 5757-5765

69. Hao T, Wu X, Xu L, Liu L, Ma W, Kuang H, Xu C. Ultrasensitive detection of prostate-specific antigen and thrombin based on goldupconversion nanoparticle assembled pyramids. Small, 2017, 13 (19): 1603944

70. Yang L, Li N, Wang K, Hai X, Liu J, Dang F. A novel peptide/ $\mathrm{Fe}_{3} \mathrm{O}_{4} @ \mathrm{SiO}_{2}$ - $\mathrm{Au}$ nanocomposite-based fluorescence biosensor for the highly selective and sensitive detection of prostate-specific antigen. Talanta, 2018, 179: 531-537

71. Xu D D, Deng Y L, Li C Y, Lin Y, Tang H W. Metal-enhanced fluorescent dye-doped silica nanoparticles and magnetic separation: A sensitive platform for one-step fluorescence detection of prostate specific antigen. Biosensors \& Bioelectronics, 2017, 87: $881-887$ 
72. Li X, Wei L, Pan L, Yi Z, Wang X, Ye Z, Xiao L, Li H W, Wang J. Homogeneous immunosorbent assay based on single-particle enumeration using upconversion nanoparticles for the sensitive detection of cancer biomarkers. Analytical Chemistry, 2018, 90(7): 4807-4814

73. Wegner $\mathrm{K}$ D, Jin $Z$, Lindén $\mathrm{S}$, Jennings $\mathrm{T}$ L, Hildebrandt $\mathrm{N}$. Quantum-dot-based Förster resonance energy transfer immunoassay for sensitive clinical diagnostics of low-volume serum samples. ACS Nano, 2013, 7(8): 7411-7419

74. Tagit O, Hildebrandt N. Fluorescence sensing of circulating diagnostic biomarkers using molecular probes and nanoparticles. ACS Sensors, 2017, 2(1): 31-45

75. Garcia-Cortes M, Encinar J R, Costa-Fernandez J M, Sanz-Medel A. Highly sensitive nanoparticle-based immunoassays with elemental detection: Application to Prostate-Specific Antigen quantification. Biosensors \& Bioelectronics, 2016, 85: 128-134

76. Zhang K, Lv S, Lin Z, Tang D. CdS:Mn quantum dot-functionalized g- $\mathrm{C}_{3} \mathrm{~N}_{4}$ nanohybrids as signal-generation tags for photoelectrochemical immunoassay of prostate specific antigen coupling DNAzyme concatamer with enzymatic biocatalytic precipitation. Biosensors \& Bioelectronics, 2017, 95: 34-40

77. Annio G, Jennings T L, Tagit O, Hildebrandt N. Sensitivity enhancement of förster resonance energy transfer immunoassays by multiple antibody conjugation on quantum dots. Bioconjugate Chemistry, 2018, 29(6): 2082-2089

78. Mattera L, Bhuckory S, Wegner K D, Qiu X, Agnese F, Lincheneau C, Senden T, Djurado D, Charbonnière L J, Hildebrandt N, Reiss P. Compact quantum dot-antibody conjugates for FRET immunoas- says with subnanomolar detection limits. Nanoscale, 2016, 8(21): 11275-11283

79. Kavosi B, Navaee A, Salimi A. Amplified fluorescence resonance energy transfer sensing of prostate specific antigen based on aggregation of CdTe QDs/antibody and aptamer decorated of AuNPs-PAMAM dendrimer. Journal of Luminescence, 2018, 204: 368-374

80. Yang T, Hou P, Zheng L L, Zhan L, Gao P F, Li Y F, Huang C Z. Surface-engineered quantum dots/electrospun nanofibers as a networked fluorescence aptasensing platform toward biomarkers. Nanoscale, 2017, 9(43): 17020-17028

81. Li X, Li W, Yang Q, Gong X, Guo W, Dong C, Liu J, Xuan L, Chang J. Rapid and quantitative detection of prostate specific antigen with a quantum dot nanobeads-based immunochromatography test strip. ACS Applied Materials \& Interfaces, 2014, 6(9): 6406-6414

82. Kong R M, Ding L, Wang Z, You J, Qu F. A novel aptamerfunctionalized $\mathrm{MoS}_{2}$ nanosheet fluorescent biosensor for sensitive detection of prostate specific antigen. Analytical and Bioanalytical Chemistry, 2015, 407(2): 369-377

83. Pei H, Zhu S, Yang M, Kong R, Zheng Y, Qu F. Graphene oxide quantum dots@silver core-shell nanocrystals as turn-on fluorescent nanoprobe for ultrasensitive detection of prostate specific antigen. Biosensors \& Bioelectronics, 2015, 74: 909-914

84. Fang B Y, Wang C Y, Li C, Wang H B, Zhao Y D. Amplified using DNase I and aptamer/graphene oxide for sensing prostate specific antigen in human serum. Sensors and Actuators. B, Chemical, 2017, 244: 928-933 